\title{
Low Temperature Specimen Preparation for Electron Tomography
}

\author{
Mary K. Morphew and Andreas Hoenger \\ Boulder Laboratory for 3D Electron Microscopy of Cells \\ Department of Molecular, Cellular and Developmental Biology \\ University of Colorado, Boulder, Colorado 80309
}

Electron tomography (ET) has allowed cell biologists to determine cellular structure at remarkable resolution $(4-6 \mathrm{~nm})$ when combined with methods of rapid freezing. The detail that is visible in these tomograms is impressive and can help characterize important facets of cell organization. The preparation of biological specimens for ET is one of the most critical steps because of the importance of reliably preserving the native state of the sample yet render it suitable for recording large series of serial tilted views in the electron microscope. Conventional fixation by immersion into chemical cross-linkers has yielded a wealth of valuable information about the organization of cells and tissues, but the time required for diffusion of these cross-linkers is relatively slow and different macromolecules may react differently to chemical fixatives (Fig. 1A). Alternatively, samples prepared for imaging in the native frozen-hydrated state don't have artifacts from chemical fixation but are challenging to serially image for ET due to their low contrast and beam sensitivity (Fig. 1C). Rapid freezing/freeze substitution fixation (RF/FSF) and plastic embedding has been a useful fusion method that has produced exciting and reproducible results (Fig. 1B). High pressure freezing vitrifies cellular samples up to $300 \mu \mathrm{m}$ thick and provides a versatile method for cryo-immobilization of diverse cellular specimens. Such samples can then be gradually dehydrated at low temperature using an organic solvent like acetone that displaces water at $-90^{\circ} \mathrm{C}$. This process involves a solid-liquid interface at which the still-frozen cellular water stabilizes biological structure until the water is replaced at low temperature. RF/FSF greatly improves the preservation of cellular and macromolecular detail over that which is seen after dehydration in a solvent concentration series at $0^{\circ} \mathrm{C}$. Chemical fixatives or stains can be dissolved in the organic solvent used for freeze-substitution, providing cross-linking and contrast enhancement to improve conditions for EM imaging. These samples can then be embedded in plastic, sectioned and imaged, either for conventional 2D methods or in $3 \mathrm{D}$ through tomographic reconstruction by recording tilt series.

Despite the many excellent studies of EM morphology after RF/FSF into acetone, samples often suffer from ice crystal damage, which is detected in the higher resolution tomographic reconstructions. While for best results the initial vitrification of samples during rapid freezing is a crucial first step, the subsequent RF/FSF process may impose its own potential for ice damage. Due to the melting temperature of acetone at $-94.9^{\circ} \mathrm{C}$, freeze substitution requires warming the specimen to $-90^{\circ} \mathrm{C}$ at the initiation of the substitution process which is significantly higher than the $-140^{\circ} \mathrm{C}$ that marks the transition between vitrified and crystalline ice [1]. What counts for pure water may not be exactly the same for a mixed protein solution as the high solute concentration of the cytoplasm may raise the phase-transition point to some extent, but likely not to reach the melting temperature of acetone. We have begun to test the merit of starting freeze substitution at lower temperatures ($140^{\circ} \mathrm{C}$ ) with a combination of low-melting solvents in an effort to prevent ice crystal formation at the onset of freeze substitution.

A further advantage of ET is the potential to map the 3D distribution of cellular organelles and 
macromolecules in relatively large volumes of biological material. Immuno-localization of antigens on the surface of sections prepared by RS/FSF is a useful method for ET, especially when serial tomograms are combined [2]. In addition, freeze-substitution based silver enhancement of Nanogold is useful for ET, particularly in cases where Nanogold is attached to endocytosed ligand which can be located during intracellular trafficking $[3,4]$.

\section{References}

[1] J. Dubochet, (2007) The physics of rapid cooling and its implications for cryoimmobilization of cells. Methods Cell Biol. 79:7-21.

[2] Donohoe et al., (2007) Identification and characterization of COPIa- and COPIb-type vesicle classes associated with plant and algal Golgi. PNAS, 104(1):163-168.

[3] MK Morphew et al., (2008) Silver enhancement of Nanogold particles during freeze substitution for electron microscopy. J Microscopy, 230:263-267.

[4] He W, et al., (2007) A freeze substitution fixation-based gold enlarging technique for EM studies of endocytosed Nanogold-labeled molecules. J Struct Biol. 160:103-13.
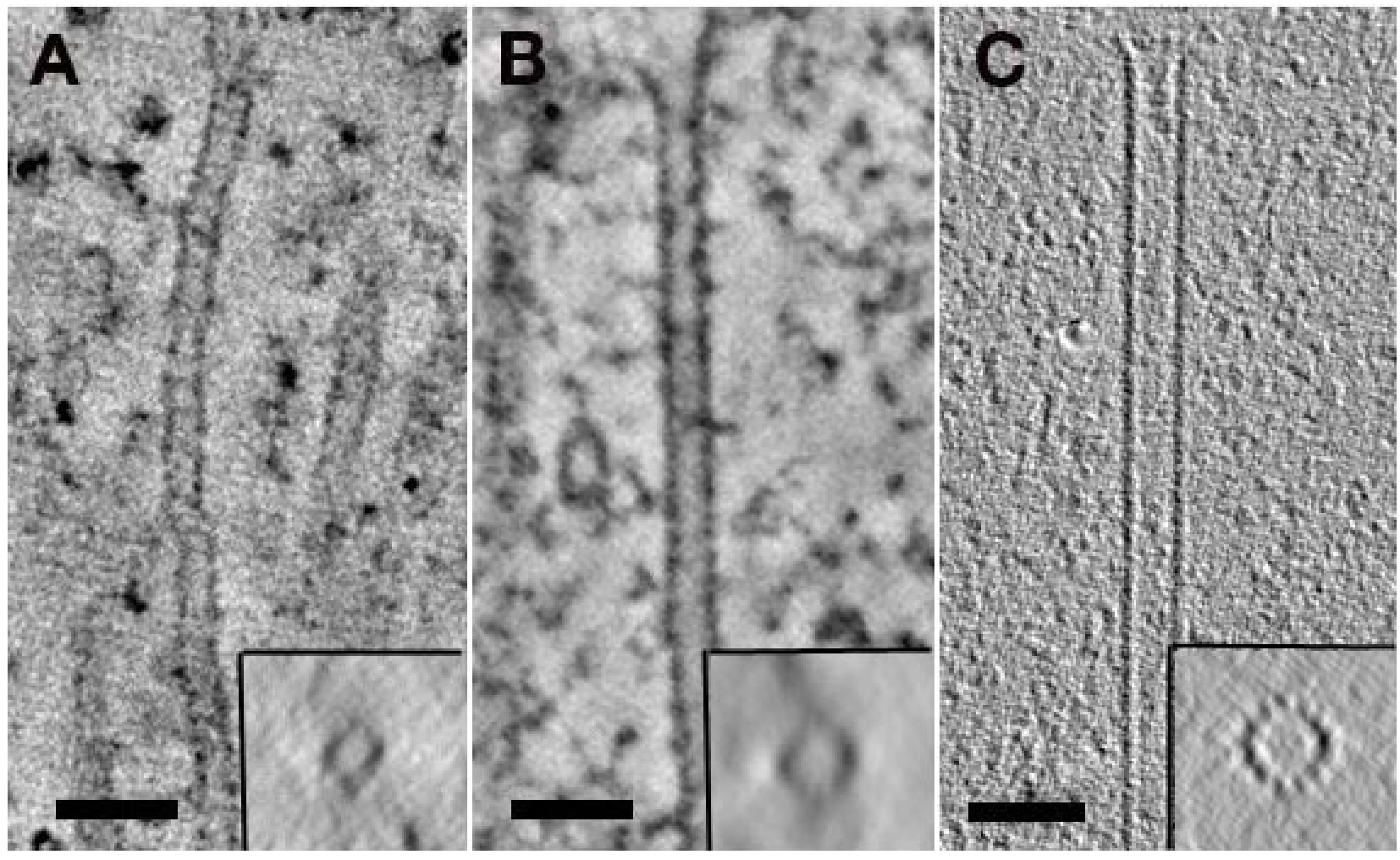

Fig.1. Comparison of microtubule structure in $7 \mathrm{~nm}$ tomographic slices from cells prepared by three different methods. A. Conventional immersion fixation and epoxy embedding. B. Rapid freezing, freeze-substitution and epoxy embedding. C. Native, frozen-hydrated, imaged in ice. Bars=50nm. 